\title{
Aortic root and valve proportions: an example of the golden ratio?
}

\author{
Nikoloz Koshkelashvili', Mauricio Jalife Bucay², Igor Goykhman³, Gregg S. Pressman ${ }^{4}$ \\ ${ }^{1}$ Department of Medicine, Division of Cardiology, Emory University, Atlanta, GA; ${ }^{2}$ Department of Internal Medicine, \\ Einstein Medical Center, Philadelphia, PA; ${ }^{3}$ Department of Radiology, Einstein Medical Center, Philadelphia, PA; \\ ${ }^{4}$ Institute for Heart and Vascular Health, Einstein Medical Center, Philadelphia, PA, USA
}

\begin{abstract}
Calcific aortic stenosis is the most common lesion requiring valve replacement. Transcutaneous procedures (TAVR) are rapidly increasing yet detailed information on aortic valve and root anatomy are sparse. This study examined gated cardiac CT scans to make observations regarding the size and proportions of the valve leaflets, sinuses of Valsalva, and sinotubular junction. One hundred and fifty gated cardiac CT scans were performed for a variety of clinical indications. Area of each cusp (short axis plane) was measured along with sinus height (from leaflet base to sinotubular junction), sinus width (from central coaptation point to outer sinus edge), annular perimeter, perimeter at midsinus level, and perimeter at the sinotubular junction. The right coronary cusp was largest, the left cusp smallest, and the noncoronary cusp intermediate in size. Each cusp was larger in men than women, even after indexing for body surface area. By contrast, indexed sinus width and height did not differ between genders. No significant differences were noted between races. Annular perimeter did not vary by age while the mid-sinus perimeter and sinotubular junction perimeter increased with age $\left(\mathrm{p}=0.01, \mathrm{r}^{2}=0.05\right.$ and $\left.\mathrm{p}=0.002, \mathrm{r}^{2}=0.07\right)$. Interestingly, the ratio of sinus height (average): annular radius was $1.69 \pm 0.18$, very close to the "golden ratio" of 1.62 found throughout the natural world. This might be important for proper vortex formation in the sinuses.
\end{abstract}

Correspondence: Gregg S. Pressman, Room 3230, Levy Building, Einstein Medical Center, 5501 Old York Rd, Philadelphia, PA 19141, USA. Fax: +1.215.456-3533. E-mail: pressmang@einstein.edu

Key words: Aortic root; aortic valve; golden ratio.

Received for publication: 23 December 2018.

Accepted for publication: 1 July 2019.

(C) Copyright N. Koshkelashvili et al., 2019

Licensee PAGEPress, Italy

Monaldi Archives for Chest Disease 2019; 89:1022

doi: 10.4081/monaldi.2019.1022

This article is distributed under the terms of the Creative Commons Attribution Noncommercial License (by-nc 4.0) which permits any noncommercial use, distribution, and reproduction in any medium, provided the original author(s) and source are credited.

\section{Introduction}

The aortic root is an elegantly designed structure that provides support for the aortic valve while minimizing stress on the valve leaflets [1-3]. It encompasses the aortic valve annulus (a virtual structure [4]), the sinuses of Valsalva, and the sinotubular junction. The sinuses allow for vortex formation which aids in aortic valve closure in diastole [5]. There are also complex interactions between the sinuses and coronary blood flow [6,7]. The advent of transcatheter aortic valve replacement (TAVR) has focused more attention on the aortic root, yet data on aortic root dimensions in the general population are sparse. The aim of this study was to make detailed measurements of the various aortic root components in a diverse clinical sample.

\section{Methods}

One hundred and fifty patients undergoing gated cardiac CT scan (inpatient or outpatient) for any clinical indication between January 2015 and December 2015 were enrolled. Demographic and clinical data were gathered from electronic medical records. Diabetes was considered present if listed as a diagnosis or based on use of diabetic medication. Similarly hypertension was deemed present based on recorded diagnosis or the use of antihypertensive medication. Coronary artery disease was considered present if listed as a diagnosis or if prior studies (e.g. stress testing or coronary angiography) so indicated, or if the patient had a history of coronary intervention or coronary bypass surgery. Chronic kidney disease was defined as an estimated glomerular filtration rate (by MDRD formula) of $<60 \mathrm{ml} / \mathrm{min} / \mathrm{m}^{2}$.

All scans were performed using Brilliance iCT (Philips Healthcare, Cleveland, OH, USA) or LightSpeed VCT (General Electric Healthcare, Milwaukee, WI, USA) scanners and were ECG gated. Thin CT slices ranging between $0.62 \mathrm{~mm}$ to 1.25 $\mathrm{mm}$ were obtained from "Synapse PACS" (Fujifilm Medical Systems USA, Inc., Stamford, CT, USA). Anatomy of the aortic valve was evaluated by previously described methods ${ }^{8}$. Briefly, in the coronal oblique view, during diastole, a plane was positioned to match the coaptation point of the cusps and placed at the level of the aortic annulus, the midpoint of the sinuses of Valsalva (at the level of cusp coaptation) and at the sinotubular junction (Figure 1). Images were carefully adjusted across cross sectional and sagittal planes for the best alignment. Cross sectional views of the aortic annulus, midpoint of sinuses and sinotubular junction were used to assess the perimeters at those levels respectively. At the level of cusp coaptation, measurements of cusp area and width were obtained. The sagittal plane was 
used to measure the height of each cusp from its base to the sinotubular junction.

All 3D reconstructions were performed utilizing an "IntelliSpace 3D" workstation (Philips Healthcare).

\section{Results}

Baseline characteristics are described in Table 1, while aortic root measurements are described in Table 2.

Of the 3 cusps, the right (RCC) was the largest, the left (LCC) was the smallest, and the non-coronary cusp (NCC) was intermediate in size. Each cusp was statistically significantly larger in men than women $(\mathrm{p}<0.0001$ for each). After indexing to body surface area (BSA) the differences in mean values between men and women remained statistically significant (Table 2) but there were large areas of overlap in each case. Sinus width and height were also greater in men than women (in each case) but these differences became nonsignificant after indexing for body surface area (BSA). The annulus perimeter was $79.3 \pm 6.8 \mathrm{~mm}$ in men $v s$ $71.6 \pm 5.9 \mathrm{~mm}$ in women $(\mathrm{p}<0.0001)$; after indexing to BSA no significant gender difference was seen. No significant differences were noted between races. Annular perimeter did not vary by age. By contrast, perimeters at the mid-sinus level and at the sinotubular junction were increased at older ages though the association was weak $(p=0.01, r$ squared $=0.05$ and $p=0.02, r$ squared $=0.07$ ). Other parameters were not associated with age except for height of the right coronary sinus which showed a weak but significant inverse correlation with age $(p=0.004, r$ squared $=0.05)$. The ratio of sinus height (average):annular radius was $1.69 \pm 0.18$.

\section{Discussion}

This investigation focused on aortic root dimensions in a diverse clinical sample. There are several observations of interest including gender differences in various parameters. All perimeters and sinus measurements were larger in men than women but these differences were no longer significant after indexing for BSA. However, mean area of the individual valve leaflets was greater in men than women and these differences remained significant after indexing for BSA. We also found differences in leaflet area between the leaflets with the left cusp being the smallest and the right being the largest. The reasons for this latter variation are unclear but may have to do with adjacent anatomic structures such as the atrial septum, aorto-mitral continuity, left atrial appendage, and pulmonary trunk. In addition, the right sinus of Valsalva is not constrained by adjacent structures and has been observed to expand more in systole than the other two sinuses [2]. Related to these size differences, it is interesting to note a previous study by our group, of 318 non-contrast CT scans (performed for any reason), wherein frequency of leaflet calcifications varied by leaflet: the left cusp most often contained calcifications (39\%) followed by the noncoronary cusp (32\%), with calcifications least often present in the right cusp (24\%) [9].

Possible associations between perimeter values and age were also explored. Perimeters at the mid-sinus level and sino-tubular junction were noted to increase with increasing age. By contrast, there was little change in annular perimeter, a finding that has been reported by others [10].

Table 1. Baseline characteristics.

\begin{tabular}{lc}
\hline Age (years) & $54.7 \pm 11.3$ \\
Male (\%) & 49 \\
\hline Body surface area $\left(\mathrm{m}_{2} / \mathrm{kg}\right)$ & $1.96 \pm 0.27$ \\
Race $(\%)$ & \\
Black & 73 \\
White & 13 \\
Hispanic & 8 \\
Other & 6 \\
\hline Hypertension (\%) & 30 \\
Diabetes mellitus (\%) & 27 \\
\hline Chronic kidney disease (\%) & 6 \\
Coronary artery disease (\%) & 18 \\
\hline
\end{tabular}
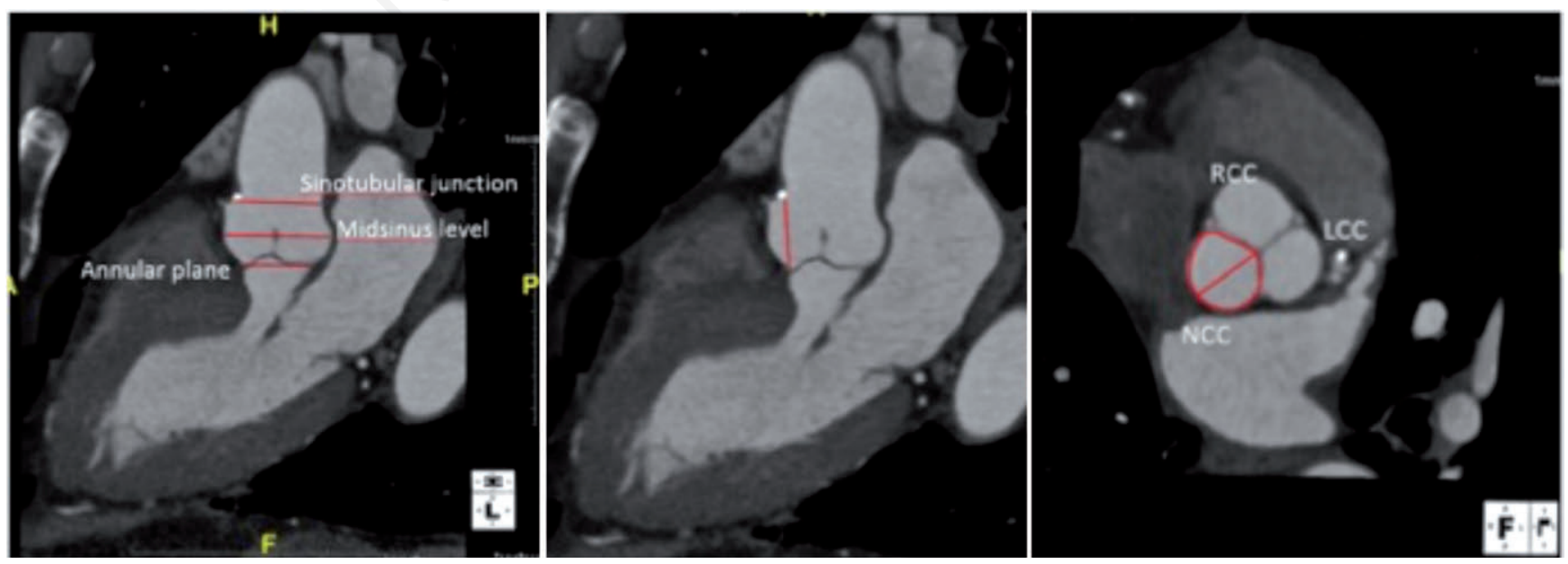

Figure 1. Left panel shows placement of the various perimeter cut planes; the center panel illustrates measurement of sinus height; the right panel illustrates measurement of cusp area and width. LCC, left coronary cusp; RCC, right coronary cusp; NCC, non-coronary cusp. 
Understanding normal aortic root dimensions and proportions are important for several reasons:

Disease can alter these relationships. This can result in hemodynamic changes and can potentially affect myocardial energetics. For example, thoracic aortic aneurysms have been reported (via in vitro models) to cause flow disruption in adjacent normal sections of the aorta, and to increase shear stresses [11]. Anatomic distortion of the aortic root can also lead to aortic regurgitation. Marfan disease would be a classic example.

Surgical reconstruction of the aortic root should aim to reproduce normal proportions as closely as possible. In this regard it is interesting to note the findings of Toninato et al. [12], who observed marked energy loss in a simulation where there were no sinuses.

Finally, we note with interest that the ratio of the sinus height to annular radius was 1.69 in this sample, very close to the "golden ratio" of 1.62 which is found throughout the natural world [13]. To our knowledge this is a new finding. The golden ratio can generate an equiangular spiral (Figure 2) which appears quite similar to the vortices that form in the sinuses of Valsalva and which were first demonstrated by Leonardo da Vinci [14]. We postulate that this ratio may be important for efficient vortex formation and optimal blood flow through the aortic root.

These findings have potential importance for understanding aortic root pathology. Determining normal ranges for its component parts allows us to define what is abnormal. We can also use this information to understand how the aortic root fails and the resultant hemodynamic/physiologic consequences. This knowledge can potentially benefit patients undergoing surgical or transcutaneous procedures of the aortic root and valve. Careful preprocedure planning is necessary to maximize the chances of a

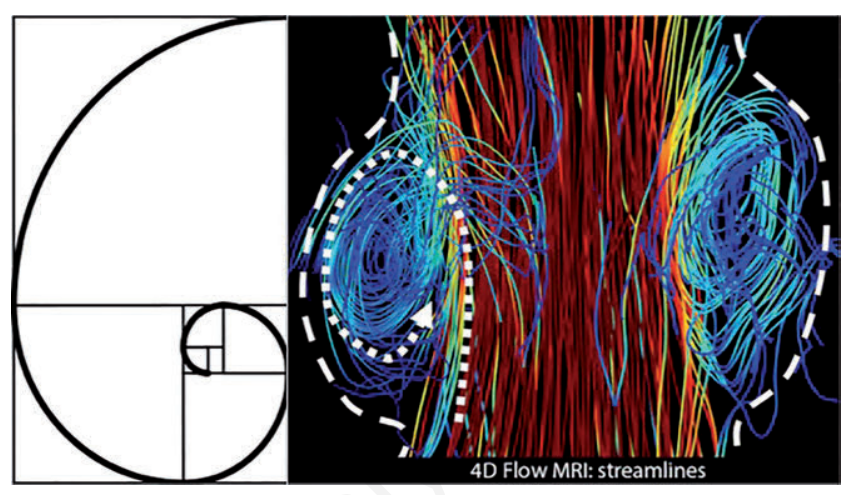

Figure 2. Left: a golden spiral - a logarithmic spiral whose growth factor is the golden ratio; the spiral gets wider (more distant from its origin) by a factor of $\mathbf{1 . 6 2}$ for every quarter turn it makes. Right: vortex flow in the sinuses of Valsalva as imaged by MRI: reproduced from Oechtering et al., J Thorac Cardiovasc Surg 2016;152:418-27, with permission.

Table 2. Aortic root measurements by gender.

\begin{tabular}{|c|c|c|c|}
\hline & Men & Women & $P$ value \\
\hline $\mathrm{RCC}\left(\mathrm{mm}^{2}\right)$ & $288.4 \pm 72.5$ & $214.3 \pm 53.2$ & $<0.0001$ \\
\hline $\operatorname{LCC}\left(\mathrm{mm}^{2}\right)$ & $252.8 \pm 58.9$ & $200.9 \pm 49.1$ & $<0.0001$ \\
\hline $\mathrm{NCC}\left(\mathrm{mm}^{2}\right)$ & $265.8 \pm 65.6$ & $213.2 \pm 52.3$ & $<0.0001$ \\
\hline Indexed RCC $\left(\mathrm{mm}^{2} / \mathrm{m}^{2}\right)$ & $141.8 \pm 38.3$ & $117.2 \pm 32.3$ & $<0.0001$ \\
\hline Indexed LCC $\left(\mathrm{mm}^{2} / \mathrm{m}^{2}\right)$ & $123.8 \pm 29.8$ & $109.2 \pm 29.3$ & 0.004 \\
\hline Indexed NCC $\left(\mathrm{mm}^{2} / \mathrm{m}^{2}\right)$ & $130.0 \pm 32.8$ & $116.5 \pm 32.8$ & 0.02 \\
\hline RCC sinus height (mm) & $21.8 \pm 3.7$ & $18.6 \pm 2.3$ & $<0.0001$ \\
\hline LCC sinus height (mm) & $21.3 \pm 3.1$ & $18.7 \pm 2.5$ & $<0.0001$ \\
\hline NCC sinus height (mm) & $22.0 \pm 3.5$ & $19.0 \pm 2.3$ & $<0.0001$ \\
\hline Indexed RCC sinus height $\left(\mathrm{mm} / \mathrm{m}^{2}\right)$ & $10.6 \pm 2.1$ & $10.2 \pm 1.7$ & 0.19 \\
\hline Indexed LCC sinus height $\left(\mathrm{mm} / \mathrm{m}^{2}\right)$ & $10.4 \pm 1.8$ & $10.2 \pm 1.8$ & 0.66 \\
\hline Indexed NCC sinus height $\left(\mathrm{mm} / \mathrm{m}^{2}\right)$ & $10.7 \pm 2.1$ & $10.4 \pm 1.6$ & 0.25 \\
\hline RCC sinus width (mm) & $18.0 \pm 2.3$ & $15.4 \pm 1.8$ & $<0.0001$ \\
\hline LCC sinus width (mm) & $16.9 \pm 2.0$ & $14.9 \pm 2.1$ & $<0.0001$ \\
\hline NCC sinus width (mm) & $18.1 \pm 2.2$ & $16.0 \pm 1.9$ & $<0.0001$ \\
\hline Indexed RCC sinus width $\left(\mathrm{mm} / \mathrm{m}^{2}\right)$ & $8.8 \pm 1.5$ & $8.4 \pm 1.4$ & 0.10 \\
\hline Indexed LCC sinus width $\left(\mathrm{mm} / \mathrm{m}^{2}\right)$ & $8.3 \pm 1.4$ & $8.2 \pm 1.4$ & 0.71 \\
\hline Indexed NCC sinus width $\left(\mathrm{mm} / \mathrm{m}^{2}\right)$ & $8.9 \pm 1.4$ & $8.8 \pm 1.5$ & 0.70 \\
\hline Annular perimeter (mm) & $79.3 \pm 6.8$ & $71.6 \pm 5.9$ & $<0.0001$ \\
\hline Mid-sinus perimeter (mm) & $113.0 \pm 9.6$ & $100.1 \pm 10.0$ & $<0.0001$ \\
\hline Sino-tubular junction perimeter (mm) & $93.1 \pm 8.9$ & $82.8 \pm 8.4$ & $<0.0001$ \\
\hline Indexed annular perimeter $\left(\mathrm{mm} / \mathrm{m}^{2}\right)$ & $38.7 \pm 4.6$ & $39.1 \pm 4.9$ & 0.63 \\
\hline Indexed mid-sinus perimeter $\left(\mathrm{mm} / \mathrm{m}^{2}\right)$ & $55.3 \pm 7.8$ & $54.8 \pm 8.3$ & 0.73 \\
\hline Indexed sino-tubular junction perimeter $\left(\mathrm{mm} / \mathrm{m}^{2}\right)$ & $45.7 \pm 7.2$ & $45.2 \pm 6.6$ & 0.64 \\
\hline
\end{tabular}


successful procedure and minimize the risk of complications. This involves detailed study of aortic root anatomy on an individual basis. But it also requires background knowledge of the spectrum of variation present in the larger population. Knowing the range of normal values for components of the aortic root can allow us to recognize potentially high-risk anatomy that falls outside these ranges.

These data come from a single institution. They were obtained from a diverse clinical sample undergoing CT examination of the chest for any reason and may not reflect dimensions in the general population. On the other hand, this sample is reflective of the type of patients encountered in every day practice and our results are therefore relevant to the clinical cardiologist. We further acknowledge that there may be racial/ethnic differences in aortic root proportions; this should be explored in future research. Our measurements might also differ from the dimensions of the "ideal" root due to the presence of hypertension, diabetes, and other comorbidities in our subjects, as well as effects of wear and tear over the decades. Perhaps the ideal situation would include a sinus height: annular radius ratio even closer to the golden ratio. In this sample of patients undergoing gated cardiac CT for a variety of clinical reasons we observed larger valve leaflet areas in men vs women, even after indexing for BSA. There was also variation in area between the individual cusps with the right being the largest and the left the smallest. With increasing age there was no change in aortic annular size while there was enlargement of the sinuses and the sinotubular junction. Interestingly the ratio of sinus height:annular radius was $1.69: 1$, very close to the "golden ratio" found throughout the natural world. This may have to do with optimal vortex formation which allows efficient flow of blood across the aortic valve and aids in valve closure.

\section{References}

1. Schoen FJ. Evolving concepts of cardiac valve dynamics: the continuum of development, functional structure, pathobiology, and tissue engineering. Circulation 2008;118:1864-1880.

2. Lansac E, Lim HS, Shomura Y, et al. Aortic root dynamics are asymmetric. J Heart Valve Dis 2005;14:400-7.
3. Berdajs D, Mosbahi S, Forro Z, et al. Numerical analysis of the 3-dimensional aortic root morphology during the cardiac cycle. Eur J Cardiothorac Surg 2016;49:1213-21.

4. Kasel AM, Cassese S, Bleiziffer S, et al. Standardized imaging for aortic annular sizing: implications for transcatheter valve selection. JACC Cardiovasc Imaging 2013;6:249-62.

5. Katayama S, Umetani N, Sugiura S, Hisada T. The sinus of Valsalva relieves abnormal stress on aortic valve leaflets by facilitating smooth closure. J Thorac Cardiovasc Surg 2008; 136:1528-35, 1535.e1.

6. Moore BL, Dasi LP. Coronary flow impacts aortic leaflet mechanics and aortic sinus hemodynamics. Ann Biomed Eng 2015;43:2231-2241.

7. Stein PD, Blick EF, Shields SK, Matta F. Sinus of Valsalva: a converging nozzle that contributes to stable flow in the coronary arteries. J Anat 2014;225:94-97.

8. Hoey ET, Ganeshan A. Multi-detector CT angiography of the aortic valve-Part 1: anatomy, technique and systematic approach to interpretation. Quant Imaging Med Surg 2014;4: 265-272.

9. Koshkelashvili N, Codolosa JN, Goykhman I, et al. Distribution of mitral annular and aortic valve calcium as assessed by unenhanced multidetector computed tomography. Am J Cardiol 2015;116:1923-27.

10. Mirea O, Maffessanti F, Gripari P, et al. Effects of aging and body size on proximal and ascending aorta and aortic arch: inner edge-to-inner edge reference values in a large adult population by two-dimensional transthoracic echocardiography. J Am Soc Echocardiogr 2013;26:419-27.

11. Numata S, Itatani $K$, Kanda $K$, et al. lood flow analysis of the aortic arch using computational fluid dynamics. Eur $\mathrm{J}$ Cardiothorac Surg 2016;49:1578-85.

12. Toninato R, Salmon J, Susin FM, et al. Physiological vortices in the sinuses of Valsalva: An in vitro approach for bio-prosthetic valves. J Biomech 2016;49:2635-43.

13. Ozturk S, Yalta K, Yetkin E. Golden ratio: A subtle regulator in our body and cardiovascular system? Int J Cardiol 2016;223:143-5.

14. Bissell MM, Dall'Armellina E, Choudhury RP. Flow vortices in the aortic root: in vivo 4D-MRI confirms predictions of Leonardo da Vinci. Eur Heart J 2014;35:1344. 